JHEPATOL-D-16-00282 R1

\title{
Electroencephalogram variability in patients with cirrhosis associates with the presence and severity of hepatic encephalopathy
}

Søren Schou Olesen ${ }^{1}$, Mikkel Gram ${ }^{1}$, Clive Douglas Jackson ${ }^{2}$, Edwin Halliday ${ }^{3}$, Thomas Holm Sandberg $^{1}$, Asbjørn Mohr Drewes ${ }^{1,4}$, Marsha Yvonne Morgan ${ }^{3}$

${ }^{1}$ Mech-Sense, Department of Gastroenterology and Hepatology, Aalborg University Hospital, Denmark

2. Department of Neurophysiology, Royal Free Hospital, Royal Free London NHS Foundation Trust, Hampstead, London, UK

3. UCL Institute for Liver and Digestive Health, Division of Medicine, Royal Free Campus, University College London, Hampstead, London, UK

4. Department of Clinical Medicine, Aalborg University, Aalborg, Denmark

\section{Corresponding author:}

Søren Schou Olesen, MD, PhD

Mech-Sense, Department of Gastroenterology \& Hepatology

Aalborg University Hospital, Hobrovej 18-22, 9100 Aalborg, Denmark

Telephone: +45 9936243, Fax: +4599326507

E-mail: sso@mech-sense.com

Electronic word count: 5647

Number of figures and tables: Figures: 1; Tables 3, Supplementary tables 1

Key-words: Biological variability; Cirrhosis; Continuous Wavelet Transform; Electroencephalogram; Hepatic encephalopathy; Sample entropy; Spectral analysis

Abbreviations: CWT, Continuous wavelet transform; EEG, electroencephalogram;

HE, hepatic encephalopathy

Conflicts of interest: None

Financial support: None 
JHEPATOL-D-16-00282 R1

\section{Authors' contributions:}

SSO: contributed to study design and data quality control; undertook the statistical analysis; interpreted the data and drafted the manuscript. MG: undertook the advanced processing of the EEG data and reviewed the manuscript. CDJ: helped create the database; undertook the original processing of the EEGs; age and gender corrected all the processed data; assisted with the statistical analysis and the revision of the manuscript. $\mathrm{EH}$ : assisted in the assessment of the patients; identified the reference population; created the original database and reviewed the manuscript. AMD: contributed to study design; assisted in the interpretation of the data and the drafting and revision of the manuscript. MYM: identified and assessed the patients; helped create the database; assisted in the interpretation of the data and the drafting and revision of the manuscript. 
JHEPATOL-D-16-00282 R1

\begin{abstract}
Background \& Aim: The outputs of physiological systems fluctuate in a complex manner even under resting conditions. Decreased variability or increased regularity of these outputs is documented in several disease states. Changes are observed in the spatial and temporal configuration of the electroencephalogram (EEG) in patients with hepatic encephalopathy (HE), but there is no information on the variability of the EEG signal in this condition. The aim of this study was to measure and characterize EEG variability in patients with cirrhosis and to determine its relationship to neuropsychiatric status.
\end{abstract}

Methods: Eyes-closed, awake EEGs were obtained from 226 patients with cirrhosis, classified, using clinical and psychometric criteria, as neuropsychiatrically unimpaired $(n=127)$ or as having minimal $(n=21)$ or overt ( $\mathrm{n}=78) \mathrm{HE}$, and from 137 healthy controls. Analysis of EEG signal variability was undertaken using continues wavelets transform and sample entropy.

Results: EEG variability was reduced in the patients with cirrhosis compared to healthy controls (coefficient of variation: $21.2 \%$ [19.3-23.4] vs. 22.4\% [20.8-24.5]; $\mathrm{p}<0.001$ ). A significant association was observed with neuropsychiatric status; EEG variability was increased in patients with minimal HE compared with their neuropsychiatrically unimpaired counterparts (sample entropy: 0.98 [0.87-1.14] vs. 0.83 [0.75-0.95]; $\mathrm{p}=0.02)$, while it was suppressed in the patients with overt HE compared to those with minimal HE (sample entropy: 0.82 [0.71-1.01] vs. 0.98 [0.87-1.14]; $\mathrm{p}=0.01$ ).

Conclusion: Variability of the EEG is associated with both the presence and severity of HE. This novel finding may provide new insights to the pathophysiology of $\mathrm{HE}$ and provide a means for monitoring patients over time. 
JHEPATOL-D-16-00282 R1

\section{Introduction}

Hepatic encephalopathy (HE) is a common and potentially disabling complication of chronic liver disease. It reflects a continuum of neuropsychiatric abnormalities ranging from discrete impairment of executive brain functions to profound coma [1]. The development of HE, compromises daily living activities and reduces patients' life quality and has a significant negative effect on survival [2-5]. Nevertheless, there is still no 'gold standard' for the diagnosis of this syndrome and in particular the diagnosis of minimal HE remains difficult to establish due to the discrete cognitive abnormalities characterising the lower end of the HE spectrum [1].

The electroencephalogram (EEG) reflects cortical neuronal activity and has been used to facilitate the diagnose of HE since the early 1950s [6]. The main electrophysiological characteristic of HE is slowing of the mean frequency from the alpha range towards the theta and delta ranges [7]. The diagnostic efficacy of the EEG, in this situation, is difficult to gauge because: (i) there is no diagnostic gold standard for comparison, and: (ii) result may vary depending on whether reports are based on visual inspection or spectral analysis. Thus, there is considerable variation in the reported performance of the EEG, with sensitivities, for the diagnosis of overt $\mathrm{HE}$, ranging from 43 to $100 \%$ and specificities ranging from 41 to $88 \%$ [7].

A number of other EEG features have been observed in patients with HE including, transient fast activities [7]; distinct topographic changes over the temporal and frontal cortical [7]; and dissociation and anteriorisation of the posterior basic rhythm with disease progression [8-11]. However, the predominant use of frequency estimates, for diagnostic purposes, has resulted in a relative disregard of these other EEG characteristics and hence little is known about their importance or diagnostic utility.

The EEG signal, like many physiological time series, is complex, as the information contained within the signal comprises a multitude of fast and slow frequencies with changing potentials. In addition, and contrary to classical concepts of physiological control, the output fluctuates in a complex manner, even in 
JHEPATOL-D-16-00282 R1

the resting state [12]. Deceased variability or increased regularity of a number of physiological rhythms has been reported in different clinical settings and associates with disease severity and negative outcomes [13]. It has been shown, for example, that the complexity of the EEG decreases up to 25 min before an epileptic seizure [14] and that the complexity of the EEG decreases in Alzheimer's disease [15].

The advent of mathematical techniques with a basis in chaos theory and nonlinear dynamics and the parallel advancements in computational methodology have facilitated the measurement and characterization of the variability and complexity of time series [16]. Continuous wavelet transform (CWT) and sample entropy are techniques, which, in addition to conventional spectral analysis, can be used to characterise variability and transient features of the EEG [10,17-19]. CWT has better time-frequency resolution than Fast Fourier Transform [19], the technique most often employed for spectral analysis of the EEG. Sample entropy can be used to investigate the temporal dynamics of the EEG; it quantifies the degree of regularity vs. the degree of unpredictability of the signal and thus provided a measure of its variability [18]. Regular time series are characterized by low sample entropy while random time series, which have greater disorder and complexity, are characterized by high sample entropy.

The aims of the present study were: 1) to characterise EEG rhythmicity and its topography based on CWT spectral analysis; 2) to characterise the variability of the EEG signal using dynamic CWT estimates and sample entropy; and, 3) to investigate possible interactions between EEG variability measures, neuropsychiatric status, and the aetiology and severity of the underlying liver disease. 
JHEPATOL-D-16-00282 R1

\section{Subjects and Methods}

\section{Study populations}

The patient population comprised of 226 patients (149 men: 77 women; mean [range] age 54.8 [26-80] years) with biopsy-proven cirrhosis recruited at the Royal Free Hospital, London between 2008 and 2012. The aetiology of the liver injury was determined using clinical, laboratory, radiological and histological variables, whilst its severity was assessed using the model for end-stage liver disease (MELD) score and Pugh modification of the Child's grading system [20]. All patients were clinically stable at the time of the study. Patients were excluded if they were under 25 or over 80 years of age; if they had suffered an episode of major hepatic decompensation within seven days of the assessment date; had hyponatraemia or renal failure; had significant cardiac or respiratory failure; insulin-dependent diabetes mellitus or non-insulin dependent diabetes with poor glycaemic control; cerebrovascular disease; epilepsy; a history of significant head injury or other conditions likely to affect cerebral function. Patients were also excluded if they had misused alcohol or drugs in the previous three months or were taking psychoactive medications, including hypnotic drugs.

The reference population of 137 healthy volunteers (73 men: 64 women: mean age 38 [17-75] years) was recruited from amongst family, friends and staff working at the Royal Free Hospital, London and individuals who had experienced an isolated episode of fainting/dizziness but in whom clinical examination, the EEG, and cerebral imaging were completely normal. None had a history of liver disease, drank alcohol in excess of $20 \mathrm{~g}$ daily, took prescription or over-the-counter medications.

The study was conducted according to the Declaration of Helsinki (Hong Kong Amendment) and Good Clinical Practice (European guidelines). The protocol was approved the Royal Free Hampstead NHS Trust Ethics Committee. All participating subjects, or their appropriately appointed guardian, provided written, informed consent. 
JHEPATOL-D-16-00282 R1

\section{Overall Study Procedures}

Each recruited subject was assessed in a single session lasting approximately two hours. All assessments, apart from the EEG, were completed, in a quiet and well-lit room. The EEGs were performed in a dedicated recording room by a trained neurophysiologist. The procedures were carried out in the same order using a standard set of instructions from scripted texts.

(i). Mental State and Psychometric Assessment: Patients were clinically assessed by two hepatologists, working independently, and their mental state classified, using West Haven Criteria [21], as either clinically unimpaired or as showing features of overt HE. Psychometric performance was assessed using the Psychometric Hepatic Encephalopathy Score (PHES) battery [22], which comprises five paper and pencil tests viz: digit symbol, number connection A and B, serial dotting, and line tracing, which has both time and error components. The PHES data were adjusted and scored using UK normative reference data [23]. Composite scores of less than two standard deviations below mean reference values were considered abnormal.

(ii). EEG Recording: Electroencephalograms (EEGs) were recorded on one of two digital EEG systems, Walter-Graphtek PL-Winsor (Walter-Graphtek GmbH, Emmendingen, Gerrmany) or MicroMed SystemPlus EVOLUTION (Micromed Sp.A., Mogliano, Veneto, Italy). Recordings were undertaken for six minutes, in a state of eyes-closed, relaxed, wakefulness, using 23 silver-silver chloride electrodes placed according to the International 10-20 System. The impedance of the electrodes was kept below $5 \mathrm{~K} \Omega$.

\section{Classification of Neuropsychiatric Status}

Neuropsychiatric status was classified, on the day of the study, as: (i) unimpaired: no clinical evidence of HE and no psychometric abnormalities; (ii) minimal HE: no clinical abnormalities but impaired psychometric performance, defined supra vide; (iii) overt HE: clinically evident, characteristic neuropsychiatric disturbances. 
JHEPATOL-D-16-00282 R1

\section{EEG Analysis}

(i). EEG pre-processing: A consecutive, 60-100 seconds of eye-closed, artefact-free recording was selected from all suitable recordings for EEG analysis. If an insufficient length of artefact-free recoding were available the selection criteria were relaxed to include sections that were artefact-free on the P3-P4 derivation.

(ii). CWT spectral and variability estimates: CWT is based on a mother wavelet function, which can be translated and dilated to calculate time-frequency coefficients. The mother wavelet function can be chosen from a set of infinite functions. For purposes of this study a complex Morlet wavelet function with a bandwidth of $10 \mathrm{~Hz}$ and a centre frequency of $1 \mathrm{~Hz}$ was used. Scales for the mother wavelet were chosen to match frequencies ranging from $1-32 \mathrm{~Hz}$ with a $0.5 \mathrm{~Hz}$ between-scale frequency interval. The wavelet coefficients were divided into standardized frequency bands viz: delta $(1-4 \mathrm{~Hz})$, theta $(4-8 \mathrm{~Hz})$, alpha $(8-12$ $\mathrm{Hz})$ and beta $(12-32 \mathrm{~Hz})$ and averaged over time; the scales contained within each frequency band were summated to provide a measure of the absolute activity. The relative activity was calculated separately for each channel by dividing individual frequency band with the summated energy for all bands and multiplying by 100. EEG variability was calculated as the Coefficient of Variation (CV) for the wavelet coefficients. Hence, for each frequency band successive epochs of $2 \mathrm{~s}$ were extracted from the CWT output and the CV was-calculated as the standard deviation of the wavelet coefficient divided by the-its mean_-of the wavelet coefficientusing Matlab 2012a (The Matworks, Inc., Natick, MA, USA). _The average CV across all frequency bands was reperted $\underline{\text { used to }}$ ebtain-provide a single measure of EEG variability.Matlab 2012a (The Matworks, Ine., Natick, MA, USA) was used for analysis.

(iii). Sample entropy: Sample entropy provides a measure of disorder or randomness and hence system complexity [18]. It is defined as the logarithmic likelihood of the repetition of patterns in a time series and estimates the probability that an epoch of window length $m$, with a degree of tolerance $r$, will be repeated at later time points. Calculation of sample entropy was undertaken using Matlab 2012a (The Matworks, Inc., 
JHEPATOL-D-16-00282 R1

Natick, MA, USA) with values for $m$ fixed at $2 \mathrm{~ms}$ and $r$ at $0.2 \mathrm{~ms}$. The average sample entropy across all frequency bands was used to provide a single measure for analysis.

\section{Data processing and statistical analysis}

The distribution of variables was tested by visual inspection of QQ-plots and the assumption of variance homogeneity was checked by the Levene`s test. The potential confounding effects of gender and age on EEG variables were assessed in the reference population using linear regression and the necessary adjustments applied to the patients' EEG variables prior to the main statistical analysis. A grand mean across all electrodes was calculated for the spectral and variability EEG measures. Differences between normally distributed EEG variables were examined by one-way ANOVA with subsequent between group comparisons performed with the Tukey HSD (Honest Significant Difference) test. Differences between non-normally distributed variables were examined using the Kruskal-Wallis test with subsequent betweengroups comparisons performed using the Mann-Whitney U or Dunn's Pairwise Comparison test with Bonferroni corrections applies for multiple comparisons._Univariate logistic regression analysis was used to examine the relationship between the aetiology and severity of the liver disease, the presence of clinically overt HE and the PHES score and the two EEG variability measures. All four variables were included in multivariate analyses. All reported $\mathrm{P}$ values were two-tailed with value less than 0.05 considered significance. The software package STATA version 11.2 (StataCorp LP, College Station, Texas) and R version 3.0.2 (CRAN.R-project.org/doc/FAQ/R-FAQ.html) were utilized. 
JHEPATOL-D-16-00282 R1

\section{Results}

\section{Patient Population}

The aetiology of the cirrhosis was alcohol in 157 (69.5\%) of the 226 patients; alcohol and hepatitis C/B virus (HCV/HBV) infection in $21(9.3 \%)$; cryptogenic in $12(5.3 \%)$; fatty liver disease in $11(4.9 \%)$; $\mathrm{HCV} / \mathrm{HBV}$ infection in eight (3.5\%); primary biliary cirrhosis in six $(2.7 \%)$; chronic active hepatitis in five (2.2\%); haemochromatosis in two (0.9\%); and 'other' in four (1.8\%). Functionally: 135 (59.7\%) of the patients were classified as Child's Grade A; 51 (22.6\%) as Child's Grade B; and $40(17.7 \%)$ as Child's Grade C (Table 1).

On the day of study: 127 (56.2\%) of the 226 patients were classified as neuropsychiatrically unimpaired, $21(9.3 \%)$ as having minimal and $78(34.5 \%)$ as having overt HE (Table 1$)$. None of the patients classified as neuropsychiatrically unimpaired or as having minimal HE had a history of previous overt $\mathrm{HE}$; $50 \%$ of those classified as having overt HE had documented evidence of previous, clinically apparent, neuropsychiatric impairment. The patients with overt HE had, as expected, significantly less functional hepatic reserve than the patients in the other two groups, evidenced by their higher mean MELD and Pugh's score; they also showed a significantly greater degree of psychometric impairment (Table 1). The distributions of patients in the alcohol-related and non alcohol-related groups were proportionate in relation to the degree of neuropsychiatric impairment (unimpaired 93 [59\%] vs. 21 [44\%], minimal HE 12 [8\%] vs. 7 [15\%] and overt HE 52 [33\%] vs. 20 [42\%]; $\mathrm{p}=0.12$ respectively).

The reference population were significantly younger than the patients; there were no significant differences in age between the patient subgroups (Table 1).

\section{Spectral EEG analysis}

A total of 226 EEGs were recorded in patients with cirrhosis and 137 in the reference population; none was discarded because of abnormal focal activity. Analysable data were available from all patient and reference EEGs. 
JHEPATOL-D-16-00282 R1

Compared to the reference population, the patients with cirrhosis had higher median (interquartile range) activities in the theta band (16.8\% [14.1-21.4] vs. 15.2\% [13.6-17.0]; $\mathrm{p}<0.0001)$ and decreased activities in the alpha band (21.4\% [16.9-26.3] vs. 23.2\% [18.0-28.9]; $\mathrm{p}=0.03)$.

A significant slowing of the EEG was observed in the patients with overt HE compared to those who were neuropsychiatrically unimpaired and those with minimal HE. This was evidenced by increased theta activity $(22.9 \%[17.1-27.6]$ vs.15.4\% [13.6-18.0] vs. $16.3 \%[13.6-17.5] \mathrm{p}<0.001)$ and decreased alpha (18.7\% [15.6-22.4] vs. 23.0\% [18.4-28.1] vs. 24.8\% [19.6-27.4]; $\mathrm{p}<0.001)$ and beta $(34.7 \%$ [27.7-42.3] vs. $39.9 \%$ [34.5-45.1] vs. 39.5\% [35.6-42.9]; $\mathrm{p}=0.001)$ activities (Table 2).

There were significant topographical differences between spectral variables in the patients with cirrhosis in relation to their neuropsychiatric status (Figure 1; Supplementary Table 1). The most prominent overall feature was anteriorisation and dissociation of the posterior basic alpha rhythm and its gradual replacement with theta activity, and later delta activity, as the degree of neuropsychiatric impairment increased. In addition, the beta activity increased symmetrically in the frontal and temporal areas in the neuropsychiatrically unimpaired patients and those with minimal HE, but was comparable to reference values in the patients with overt HE.

Beta activities were significantly higher in the patients with alcohol-related cirrhosis than in their nonalcohol-related counterparts $(39.6 \%[34.5-44.5]$ vs. 34.4\% [29.2-40.6]; $(\mathrm{p}<0.001)$, otherwise there were no significant differences in frequency estimates between patient subgroups in relation to disease aetiologyThis difference remained significant after stratification of patients by disease aetiology and neuropsychiatric status (Table 3).

EEG variability analysis 


\section{JHEPATOL-D-16-00282 R1}

Coefficient of variation: The median average $\mathrm{CV}$ was significantly reduced in the patients with cirrhosis compared to the healthy controls $(21.2 \%$ [19.3-23.4] vs. 22.4\% [20.8-24.5]; p<0.001) (Table 2). This reflected a reduction in median EEG variability in the patients with overt HE. The median average CV was significantly reduced in the patients with alcohol-related cirrhosis compared to the healthy controls but the results in the non-alcohol-related cirrhosis and the healthy controls were comparable (Table 3).

Sample entropy: There was no significant difference in the median average sample entropy between the patients with cirrhosis and the healthy controls (0.84 [0.74-1.00] vs.0.91 [0.78-1.00]; $\mathrm{p}=0.1)$. However, median average sample entropy was significantly increased in the patients with minimal HE $(0.98[0.87-$ 1.14]) compared to both the unimpaired patients $(0.83[0.75-0.95] ; \mathrm{p}=0.02)$, and the patients with overt HE (0.82 [0.71-1.01] $\mathrm{p}=0.01$ ) (Table 2). Median average sample entropy was reduced in patients with non alcohol-related cirrhosis compared to both the reference population $(0.77$ [0.67-0.95] vs. 0.91 [0.78-1.00]; $\mathrm{p}=0.01)$ and the patients with alcohol-related cirrhosis $(0.77[0.67-0.95]$ vs. $0.85[0.77-1.01] ; \mathrm{p}=0.02)$

\section{(Table 3).}

Associated variables: The presence of clinical HE was significantly associated with average CV on univariate analysis (coefficient $=0.002 ; \mathrm{p}=0.019$ ) while the aetiology of the liver disease was significantly associated with average sample entropy (coefficient $=-0.099 ; \mathrm{p}=0.008$ )._Multivariate analysis confirmed the independence and significance of these associations viz. average $\mathrm{CV}$ and the presence of clinical $\mathrm{HE}$ (coefficient $=0.003 ; \mathrm{p}=0.007$ ); aetiology of the liver disease and sample entropy (coefficient=-0.095; $\mathrm{p}=0.011)$. 
JHEPATOL-D-16-00282 R1

\section{Discussion}

Variability of the EEG was identified, in this study, as a novel feature associated with both the presence and severity of HE; increased variability was observed in patients with minimal HE, whereas in patients with overt HE the EEG there was a loss of variability. The relationship of the EEG variability was more complex; the average CV was significantly reduced in patients with alcohol-related cirrhosis whereas the average sample entropy was significantly reduced in the patients with non-alcohol-related cirrhosis; multivariate analysis confirmed that non-alcohol-related cirrhosis was significantly and independently associated with sample entropy but did not confirm the independence of the relationship between alcoholrelated cirrhosis and CV. In addition, and in agreement with other studies, the presence of HE was characterised by slowing of the brain rhythmicity and anteriorisation of the posterior basal rhythm.

\section{Spectral EEG estimates}

Slowing of the EEG with anteriorisation of the posterior basal rhythm was observed with increasing severity of neuropsychiatric impairment, confirming previous reports based on visual, semi-quantitative and automated EEG analysis [11,24-27]. However although this finding is characteristic of HE, it is not specific as it is also seen in other conditions of reduced vigilance e.g. in the transitional wake/sleep phase [28].

In addition to the generalized slowing of the EEG there was evidence of an increase in fast beta activity in the patients with alcohol-related disease. Increased beta power has been documented in the resting EEG in 30 to $50 \%$ of alcohol dependent individuals $[2,32]$, and has been attributed to changes in gamma aminobutyric acid (GABA) neurotransmission. Variations in the GABA alpha-2 gene (GABRA2) are strongly associated with the beta frequency of the human electroencephalogram (EEG) [29]. Genetic studies in humans have provided evidence of involvement of the GABA system in the susceptibility to develop alcohol dependence through an affect on the level of neural excitability[30]. However, alcohol per se modulates GABAergic neurotransmission resulting in an increase in the beta activity in the EEG. Thus, 


\section{JHEPATOL-D-16-00282 R1}

the increase in beta activity in the EEG in these patients may represent either a trait marker of a dysfunctional gamma aminobutyric acid (GABA) system or a state marker of ongoing alcohol misuse [31,32]. None of the patients with alcohol-related cirrhosis in the present study had misused alcohol for a minimum of three months before the study. Equally the increase in the beta activity could not be ascribed to an excess of patients with minimal $\mathrm{HE}$ within this group as the distribution of patients by neuropsychiatric status did not differ by disease aetiology. Thus, the increase in beta activities observed in patients with alcohol-related cirrhosis most likely represents a trait marker of dysfunctional GABAergic neurotransmission. This, then is an important confounder which should be taken into account when interpreting the EEG in patients being screened for minimal HE.

In patients with cirrhosis, spectral estimates of the EEG have traditionally been applied for diagnostic purposes based on somewhat artificial derived thresholds [11,24,27]. However, the neuropsychiatric changes associated with HE are much more likely to represent a continuum with no natural thresholds or stages [1]. _Thus, use of simple diagnostic thresholds may result in over- or under diagnosis of the condition and should be applied and interpreted with caution. In the present study a descriptive analysis of EEG was adopted to identify distinct electrophysiological signatures of the brain dysfunction, rather than focusing on thresholds for diagnostic purposes. _The agreement between the EEG characteristics and patients categorized using conventional clinical and psychometric criteria was not absolute, which was to be expected as each modality accesses different domains of cerebral activity. The findings in the present study are echoed in a recent study of brain networks in patients with cirrhosis using functional magnetic resonance imaging and graph theoretical analysis, which showed that changes in cerebral networks were evident before the advent of either clinical changes suggestive of HE or disturbed psychometric performance [33]. Taken together, the newly derived EEG parameters [mym1]may identify abnormal cortical processing at an earlier stage than current available psychometric methods and, as such, they may be useful for its earlier identification. -Whether such 'early identification', translates to better prognostic accuracy and improved patient outcome needs to be established in prospective studies. 


\section{EEG variability}

Increased sample entropy of the EEG was observed in patients with minimal HE. This has not previously been reported and, as such, may provide a new marker of brain dysfunction in this patient population. In early studies, based on subjective analysis of the EEG, `low grade HE` was characterised by transient bursts of slow wave oscillations superimposed on the normal alpha dominated brain rhythm [6,34]. The increased sample entropy observed in patients with minimal HE, in the present study, may comprise an objective correlate of this early observation. Accordingly, in the transition from an unimpaired mental state to overt HE the physiological brain rhythm, dominated by alpha activity, is gradually replaced by slowed brain oscillations typically within the theta frequency range $(4-8 \mathrm{~Hz})_{-}[27,35]$. It could be speculated that bursts of theta activity in the early stages of HE interrupts the stability of the physiological alpha rhythm and thereby increases sample entropy of the EEG. In keeping with this, variability would be expected to decrease in patients with overt HE and acempanying slowing ofas -brain rhythmicity slows, which is in agreement with the findings in the present studypresent findings.

Increased bursts of theta activity in patients with cirrhosis may represent a neurophysiological hallmark of abnormal thalamocortical connectivity as suggested by in vitro and clinical studies of neurological and neuropsychiatric disorders [36,37]. Hence, increased low-frequency theta activities, in conjunction with a widespread and marked increase of coherence among high- and low-frequency oscillations, have been described in patients with neurogenic pain, tinnitus, Parkinson's disease, and depression [37]. Interestingly, central lateral thalamotomy normalise the EEG and symptoms in patients with neurogenic pain, which underlines the importance of abnormal thalamocortical interplay in these patients [38]. However, further studies are needed to understand the importance of thalamocortical dysrhythmia in the context of the neuropsychological abnormalities that arise in patients with cirrhosis.

The pathophysiology underlying the changes in EEG variability and its association with HE were not addressed in the present study. Suppressed dynamics of other biological systems e.g. heart rate variability 
JHEPATOL-D-16-00282 R1

are associated with disorders of neuropsychiatric dysfunction including HE [39-42]. Interestingly, the change in heart rate variability, and the association with HE observed in patients with cirrhosis, was related to an overproduction of inflammatory cytokines, thus suggesting a dysregulatedderegulated inflammatory response as a common pathogenic mechanism [39]. Future studies are needed to understand the complex interactions across biological systems in patients with cirrhosis and to further characterise their mediators.

\section{Study strengths and limitations}

This study was undertaken in a large group of well-characterized patients with cirrhosis and varying degrees of HE. The large sample size allowed for detailed stratification and analysis of factors associated with changes in EEG estimates and, in addition to neuropsychiatric status, a number of other possible confounding variables including age, gender and the severity and aetiology of the liver injury - albeit the absolute contributions of these factors were minor compared with the contribution from the alterations in neuropsychiatric status.

The loss of variability in physiological systems is a negative predictor of outcome [13, 40-42]. However, although the presence of overt HE was shown to be associated with a significant loss of EEG variability the association with disease outcome was not explored in the present study.

\section{Conclusions}

Electroencephalogram variability is suppressed in patients with cirrhosis and overt HE, while patients with minimal HE are characterized by increased EEG variability. These novel findings may represent distinct markers of brain dysfunction in patients with cirrhosis and provide new insight to the intricate brain dysfunction complicating this entity. 
JHEPATOL-D-16-00282 R1

\section{References}

[1] Bajaj JS, Wade JB, Sanyal AJ. Spectrum of neurocognitive impairment in cirrhosis: Implications for the assessment of hepatic encephalopathy. Hepatology 2009;50:2014-2021.

[2] Bustamante J, Rimola A, Ventura PJ, Navasa M, Cirera I, Reggiardo V, et al. Prognostic significance of hepatic encephalopathy in patients with cirrhosis. J Hepatol 1999;30:890-895.

[3] Amodio P, Del Piccolo F, Marchetti P, Angeli P, Iemmolo R, Caregaro L, et al. Clinical features and survival of cirrhotic patients with subclinical cognitive alterations detected by the number connection test and computerized psychometric tests. Hepatology 1999;29:1662-1667.

[4] Groeneweg M, Quero JC, De Bruijn I, Hartmann IJC, Essink-Bot ML, Hop WCJ, et al. Subclinical hepatic encephalopathy impairs daily functioning. Hepatology 1998;28:45-49.

[5] Bajaj J. Minimal hepatic encephalopathy matters in daily life. World J Gastroenterol 2008;14:3609_ 3615.

[6] Foley JM, Watson CW, Adams RD. Significance of the electroencephalographic changes in hepatic coma. Trans Am Neurol Assoc 1950;51:161-165.

[7] Montagnese S, Amodio P, Morgan MY. Methods for diagnosing hepatic encephalopathy in patients with cirrhosis-: a multidimensional approach 2004;19:281-312.

[8] Sagal T, Gimeno V, Calzada MD De, Casellas F, Maci D, Soriano MV. Brain mapping analysis in patients with hepatic encephalopathy 1990;2:221-228.

[9] Kullmann F, Hollerbach S, Lock G, Holstege A, Dierks T, Schölmerich J. Brain electrical activity mapping of EEG for the diagnosis of (sub) clinical hepatic encephalopathy in chronic liver disease Eur J Gastroenterol Hepatol 2001;13:513-522.

[10] Olesen SS, Graversen C, Hansen TM, Blauenfeldt RA, Hansen JB, Steimle K, et al. Spectral and 
JHEPATOL-D-16-00282 R1

dynamic electroencephalogram abnormalities are correlated to psychometric test performance in hepatic encephalopathy. Scand J Gastroenterol 2011;46:988-996.

[11] Montagnese S, Jackson C, Morgan MY. Spatio-temporal decomposition of the electroencephalogram in patients with cirrhosis. J Hepatol 2007;46:447-458.

[12] Lipsitz LA, Goldberger AL. Loss of 'complexity' and aging: potential applications of fractals and chaos theory to senescence. JAMA 1992;267:1806-1809.

[13] Goldberger AL, Peng C-K, Lipsitz LA. What is physiologic complexity and how does it change with aging and disease? Neurobiol Aging 2002;23:23-26.

[14] Elger CE, Lehnertz K. Seizure prediction by non-linear time series analysis of brain electrical activity. Eur J Neurosci 1998;10:786-789.

[15] Besthorn C, Sattel H, Geiger-Kabisch C, Zerfass R, Förstl H. Parameters of EEG dimensional complexity in Alzheimer's disease. Electroencephalogr Clin Neurophysiol 1995;95:84-89.

[16] Seely AJE, Macklem PT. Complex systems and the technology of variability analysis. Crit Care 2004;8:R367-384.

[17] Durka PJ. From wavelets to adaptive approximations: time-frequency parametrization of EEG. Biomed Eng Online 2003;2:1.

[18] Richman JS, Moorman JR. Physiological time-series analysis using approximate entropy and sample entropy. Am J Physiol Heart Circ Physiol 2000;278:H2039-2049.

[19] Akin M. Comparison of wavelet transform and FFT methods in the analysis of EEG signals. J Med Syst 2002;26:241-247.

[20] Pugh RNH, Murray-Lyon IM, Dawson JL, Pietroni MC, Williams R. Transection of the oesophagus for bleeding oesophageal varices. Br J Surg 1973;60:646-649. 
JHEPATOL-D-16-00282 R1

[21] Conn HO, Leevy CM, Vlahcevic ZR, Rodgers JB, Maddrey WC, Seeff L, et al. Comparison of lactulose and neomycin in the treatment of chronic portal-systemic encephalopathy. A double blind controlled trial. Gastroenterology 1977;72:573-583.

[22] Weissenborn K, Ennen JC, Schomerus H, Rückert N, Hecker H. Neuropsychological characterization of hepatic encephalopathy. J Hepatol 2001;34:768-773.

[23] Marks M, Jackson C, Montagnese S, Jenkins C, Head I, Morris R, et al. Derivation of a normative UK database for the psychometric hepatic encephalopathy score (PHES): confounding effect of ethnicity and test scoring. J Hepatol 2008;48 (Suppl_2):S119, 300.

[24] Van der Rijt CC, Schalm SW, De Groot GH, De Vlieger M. Objective measurement of hepatic encephalopathy by means of automated EEG analysis. Electroencephalogr Clin Neurophysiol 1984;57:423-426.

[25] Quero JC, Hartmann IJC, Meulstee J, Hop WCJ, Schalm SW. The diagnosis of subclinical hepatic encephalopathy in patients with cirrhosis using neuropsychological tests and automated electroencephalogram analysis. Hepatology 1996;24:556-560.

[26] Amodio P, Del Piccolo F, Pettenò E, Mapelli D, Angeli P, Iemmolo R, et al. Prevalence and prognostic value of quantified electroencephalogram (EEG) alterations in cirrhotic patients. $\mathrm{J}$ Hepatol 2001;35:37-45.

[27] Marchetti P, D'Avanzo C, Orsato R, Montagnese S, Schiff S, Kaplan PW, et al. Electroencephalography in patients with cirrhosis. Gastroenterology 2011;141:1680-1689.

[28] Broughton R, Hasan J. Quantitative topographic electroencephalographic mapping during drowsiness and sleep onset. J Clin Neurophysiol 1995;12:372-386.

[29] Lydall GJ, Saini J, Ruparelia K, Montagnese S, McQuillin A, Guerrini I, et al. Genetic association study of GABRA2 single nucleotide polymorphisms and electroencephalography in alcohol 
JHEPATOL-D-16-00282 R1

dependence. Neurosci Lett 2011;500:162-166.

[30] Saletu-Zyhlarz GM, Arnold O, Anderer P, Oberndorfer S, Walter H, Lesch OM, et al. Differences in brain function between relapsing and abstaining alcohol-dependent patients, evaluated by EEG mapping. Alcohol Alcohol 2004;39:233-240.

[31] Anstee QM, Knapp S, Maguire EP, Hosie AM, Thomas P, Mortensen M, et al. Mutations in the Gabrb1 gene promote alcohol consumption through increased tonic inhibition. Nat Commun 2013;4:2816.

[32] Bauer LO. Predicting relapse to alcohol and drug abuse via quantitative electroencephalography. Neuropsychopharmacology 2001;25:332-340.

[33] Jao T, Schröter M, Chen C-L, Cheng Y-F, Lo C-YZ, Chou K-H, et al. Functional brain network changes associated with clinical and biochemical measures of the severity of hepatic encephalopathy. Neuroimage 2015;122:332-344.

[34] Parsons-Smith BG, Summerskill WH, Dawson AM, Sherlock S. The electroencephalograph in liver disease. Lancet 1957;273:867-871.

[35] Amodio P, Orsato R, Marchetti P, Schiff S, Poci C, Angeli P, et al. Electroencephalographic analysis for the assessment of hepatic encephalopathy: comparison of non-parametric and parametric spectral estimation techniques. Neurophysiol Clin 2009;39:107-115.

[36] Llinás R, Jahnsen H. Electrophysiology of mammalian thalamic neurones in vitro. Nature $1982 ; 297: 406-408$.

[37] Llinás RR, Ribary U, Jeanmonod D, Kronberg E, Mitra PP. Thalamocortical dysrhythmia: A neurological and neuropsychiatric syndrome characterized by magnetoencephalography. Proc Natl Acad Sci USA 1999;96:15222-15227.

[38] Sarnthein J, Stern J, Aufenberg C, Rousson V, Jeanmonod D. Increased EEG power and slowed 
JHEPATOL-D-16-00282 R1

dominant frequency in patients with neurogenic pain. Brain 2006;129:55-64.

[39] Mani AR, Montagnese S, Jackson CD, Jenkins CW, Head IM, Stephens RC, et al. Decreased heart rate variability in patients with cirrhosis relates to the presence and degree of hepatic encephalopathy. Am J Physiol Gastrointest Liver Physiol 2009;296:G330-338.

[40] Kim CK, McGorray SP, Bartholomew BA, Marsh M, Dicken T, Wassertheil-Smoller S, et al. Depressive symptoms and heart rate variability in postmenopausal women. Arch Intern Med 2005;165:1239-1244.

[41] Kim DH, Lipsitz LA, Ferrucci L, Varadhan R, Guralnik JM, Carlson MC, et al. Association between reduced heart rate variability and cognitive impairment in older disabled women in the community: Women's Health and Aging Study I. J Am Geriatr Soc 2006;54:1751-1757.

[42] Vigo DE, Nicola Siri L, Ladrón De Guevara MS, Martínez-Martínez JA, Fahrer RD, Cardinali DP, et al. Relation of depression to heart rate nonlinear dynamics in patients $>$ or $=60$ years of age with recent unstable angina pectoris or acute myocardial infarction. Am J Cardiol 2004;93:756-760. 
Table 1: Demographic and assessment variables in the reference and patient populations, by neuropsychiatric status

\section{Study population}

(n)

\section{Reference population (137)}

All patients with cirrhosis: (226)

Unimpaired (127)

Minimal HE (21)

Overt HE (78)

$\begin{gathered}\text { Age } \\ (\mathbf{y r})\end{gathered}$
$39.2 \pm 16.0$
$(17-54)$
$54.8 \pm 10.0^{\wedge \wedge \wedge}$
$(26-80)$
$54.1 \pm 9.5^{\wedge \wedge}$
$(32-80)$
$56.1 \pm 7.8^{\wedge \wedge}$
$(42-71)$
$55.5 \pm 11.2^{\wedge \wedge}$
$(26-78)$

\section{Gender}

(\% men)

MELD score

53

$66^{\wedge}$

$12.0 \pm 5.1$
$(6-34)$
$34.5 \pm 13.7$
$(0: 80)$

65

$10.4 \pm 4.1$

(6-30)

$42.2 \pm 10.7$
$(21-80)$

67

$10.3 \pm 2.7$

$25.0 \pm 9.9^{* * * *}$

$$
(0-40)
$$

$68^{\wedge}$
$24.4 \pm 10.3^{* * *}$
$56.1 \pm 49.7$
$(16: 480)$

$37.1 \pm 13.7$

(16-87)

\section{NCTA}

(s)
$67.4 \pm 25.3^{* * *}$
(36-148)
$83.9 \pm 72.9^{* * *}$

(24-480)

\section{NTCB}

(s)

\section{SD}

(s)
LTT

(s)

LTE

(n errors)

$151.4 \pm 105.7$
$(22: 480)$
$98.7 \pm 55.5$
$(22-400)$
$213.2 \pm 116.6^{* * *}$
$(73-480)$
$220.7 \pm 116.6^{* * *}$
$(55-480)$

$220.7 \pm 116.6$
$(55-480)$

$70.6 \pm 37.2$
$(29: 332)$
$54.6 \pm 17.2$
$(29-111)$
$77.3 \pm 21.3^{* * *}$
$(48-138)$
$94.8 \pm 49.2^{* * * *}$

(35-332)

$$
\begin{gathered}
102.4 \pm 56.2 \\
(25: 431)
\end{gathered}
$$

$82.2 \pm 35.1$

$$
\text { (25-213) }
$$

$117.4 \pm 43.4^{* * * *}$

(67-230)

$131.4 \pm 71.5^{* * * *}$

(39-431)

$$
\begin{gathered}
64.6 \pm 61.8 \\
(0: 389) \\
48.9 \pm 40.5 \\
(2-197) \\
71.8 \pm 63.5 \\
(5-204) \\
88.2 \pm 80.2^{* * *} \\
(0-389)
\end{gathered}
$$

PHES

score $^{\&}$

$-1.5 \pm 1.5$

$-0.6 \pm 0.9$

$(-2:+2)$

$-2.6 \pm 0.5^{* * *}$

$(-4:-2)$

$-2.8 \pm 1.4^{* * *}$ $(-7: 0)$

Data are expressed as mean $\pm 1 \mathrm{SD}$ (range) or absolute number $(\%)$

HE: hepatic encephalopathy; MELD: model for end-stage liver disease; NCT-A/B, Number Connection Tests A/B; DS: Digit Symbol Test; LT-T/E: Line Tracing TestTime/Error; SD: Serial Dotting Test; PHES Psychometric Hepatic Encephalopathy Score

\&PHES score range for UK identification cohort is standardized so the original normative dataset had a mean of zero and a SD of one

Significance of the difference between the reference population and the various patient subgroups; ${ }^{\wedge} \mathrm{p}<0.05 ;{ }^{\wedge \wedge} \mathrm{p}<0.01 ; \wedge \wedge \wedge \mathrm{p}<0.001 ; \wedge \wedge \wedge \wedge p<0.0001$

Significance of the difference between unimpaired patients and those with minimal/overt HE; $* \mathrm{p}<0.05 ; * * \mathrm{p}<0.01 ; * * * \mathrm{p}<0.001 ; * * * * \mathrm{P}<0.0001$

Significance of the difference between patients with minimal and overt HE; \# $\mathrm{p}<0.05$; \#\# $\mathrm{p}<0.01$; \#\# $\mathrm{p}<0.001$; \#\#\# $\mathrm{p}<0.0001$ 
Table 2: Spectral EEG indices, coefficient of variation and sample entropy in the reference population and patients with cirrhosis,

by neuropsychiatric status

\section{Study Population}

(n)

Reference population (137)

All patients with cirrhosis: (226)

Unimpaired (127)

Minimal HE (21)

Overt HE (78)
Delta

(\%)

$20.4(17.6-24.8) \quad 15.2(13.6-17.0)$

$19.8(17.0-23.5) \quad 16.8(14.1-21.4)^{\wedge \wedge \wedge}$

$19.6(16.8-22.8) \quad 15.4(13.6-18.0)$

$19.8(16.5-22.9) \quad 16.3(13.6-17.5)$

20.7 (17.9-27.5)

Theta

(\%)
Alpha

(\%)

$23.2(18.0-28.9)$

$21.4(16.9-26.3)^{\wedge}$

$23.0(18.4-28.1)$

$24.8(19.6-27.4)$

$22.9(17.1-27.6)^{\wedge \wedge \wedge * * * * \# \# \# ~}$

$18.7(15.6-22.4)^{\wedge \wedge \wedge * * * \#}$

Beta

(\%)

$39.5(34.7-43.7)$

$38.4(32.9-44.5)$

$39.9(34.5-45.1)$

$39.5(35.6-42.9)$

$34.7(27.7-42.3)^{\wedge * * * *}$
CV

$(\%)$

Sample Entropy

$0.91(0.78-1.00)$

$0.84(0.74-1.00)$

$0.83(0.75-0.95)$

$0.98(0.87-1.14)^{*}$

$20.3(18.4-22.2)^{\wedge \wedge \wedge * *} \quad 0.82(0.71-1.01)^{\# \#}$

Data are expressed as median (interquartile range)

Dunn's Pairwise Comparison test with the Bonferroni corrections for multiple comparisons:

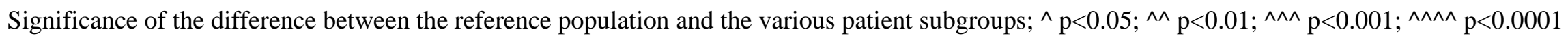

Significance of the difference between unimpaired patients and those with minimal/overt HE; $* \mathrm{p}<0.05 ; * * \mathrm{p}<0.01 ; * * * \mathrm{p}<0.001 ; * * * * \mathrm{P}<0.0001$

Significance of the difference between patients with minimal and overt HE; \# $\mathrm{p}<0.05$; \#\# $\mathrm{p}<0.01$; \#\#\# $\mathrm{p}<0.001$; \#\#\# $\mathrm{p}<0.0001$ 
JHEPATOL-D-16-00282 R1

Table 3: Spectral EEG indices, coefficient of variation and sample entropy in the reference population and patients with cirrhosis,

\begin{tabular}{|c|c|c|c|c|c|c|}
\hline \multicolumn{7}{|c|}{$\begin{array}{c}\text { Study population } \\
\text { (m) }\end{array}$} \\
\hline \multicolumn{7}{|l|}{$\begin{array}{c}\text { Delta } \\
(\%)\end{array}$} \\
\hline \multicolumn{7}{|l|}{$(\%)$} \\
\hline \multicolumn{6}{|l|}{ Alpha } & $(\%)$ \\
\hline \multicolumn{7}{|l|}{$(\%)$} \\
\hline \multicolumn{6}{|l|}{ CV } & $(\%)$ \\
\hline \multicolumn{7}{|l|}{ Sample Entropy } \\
\hline $\begin{array}{l}\text { Reference population (137) } \\
\text { Alcohol related cirrhosis (157) }\end{array}$ & $20.4(17.6-24.8)$ & $15.2(13.6-17.0)$ & $23.2(18.0-28.9)$ & $39.5(34.7-43.7)$ & $22.4(20.8-24.5)$ & $0.91(0.78-1.00)$ \\
\hline Unimpaired (93) & $19.8(16.9-22.7)$ & $15.5(13.6-17.8)$ & $22.9(18.1-27.0)$ & $39.7(35.3-45.6)$ & $21.6(19.9-23.4)$ & $0.84(0.77-0.95)$ \\
\hline Minimal HE (12) & $21.1(16.0-25.1)$ & $15.5(13.3-17.0)$ & $21.5(16.8-27.6)$ & $40.6(38.6-45.5)$ & $22.2(19.2-24.6)$ & $1.01(0.90-1.15)$ \\
\hline Overt HE (52) & $19.1\left(\begin{array}{ll}17.6 & 23.0\end{array}\right)$ & $21.6(17.0-25.9) \wedge \wedge \wedge \wedge$ & $18.3\left(\begin{array}{lll}15.4 & 22.7\end{array}\right) \wedge \wedge \wedge$ & $36.8(31.0-43.0)$ & $20.3(18.3-22.3) \wedge \wedge \Lambda$ & $0.84(0.75-1.03)$ \\
\hline Non-alcehol-related cirrhesis (48) & & & 24 & & & \\
\hline
\end{tabular}


JHEPATOL-D-16-00282 R1

\begin{tabular}{|c|c|c|c|c|c|c|}
\hline Unimpaired (21) & $19.6\left(\begin{array}{ll}15.7 & 22.8\end{array}\right)$ & $15.5\left(\begin{array}{ll}14.1 & 18.3\end{array}\right)$ & $25.3(20.9-31.8)$ & $35.1(32.3-42.9)$ & $22.1(20.3 \quad 25.9)$ & $0.78\left(\begin{array}{ll}0.69 & 0.92) *\end{array}\right.$ \\
\hline Minimal HE (7) & $19.8(17.7-22.4)$ & $19.0(15.5-23.2)$ & $24.8(19.6-27.1)$ & $35.5(33.0-40.2)$ & $24.0(20.4-29.5)$ & $0.90(0.73-1.00)$ \\
\hline Overt HE (20) & $27.1(18.3-31.6) *$ & $25.8(17.6-31.1)^{\wedge \wedge \wedge \Lambda}$ & $19.6(16.6-23.4)$ & $29.2(23.9-38.2) \wedge \wedge \wedge *$ & $21.3(19.0-22.9)$ & $0.76(0.64-0.98)$ \\
\hline
\end{tabular}

by aetiology of liver disease and neuropsychiatric status

Data are expressed as median (interquartile range)

\&Patients with alcohol-related cirrhosis and co-morbid Hepatitis B or Hepatitis C infection were omitted from the analysis (n=21)

Dunn's Pairwise Comparison test with the Bonferroni corrections for multiple comparisens:

Significance of the difference between the reference population and the various patient subgreups; $\wedge p<0.05 ; \wedge \wedge p<0.01 ; \wedge \wedge \wedge p<0.001 ; \wedge \wedge \wedge \wedge p<0.0001$

Mann-Whitney Utest:

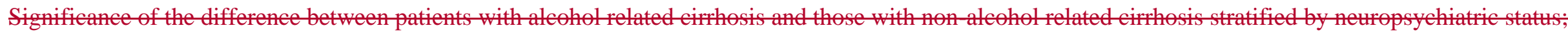
$* \mathrm{p}<0.05 ; * * \mathrm{p}<0.01 ; * * * \mathrm{p}<0.001 ; * * * * \mathrm{p}<0.0001$ 
JHEPATOL-D-16-00282 R1

\section{Legends to Figures}

Figure 1. Average head maps showing the spatial distributions of spectral EEG indices in the reference population $(n=137)$ and in the patients with cirrhosis $(n=226)$, by neuropsychiatric status.

The most prominent feature is the gradual loss of the alpha rhythm, as the degree of neuropsychiatric impairment increases, and its replacement with theta and subsequently delta activity. In addition, the beta activity increases symmetrically in the frontal and temporal areas in the neuropsychiatrically unimpaired patients and in those with minimal HE, but this increase is attenuated in patients with overt HE. 


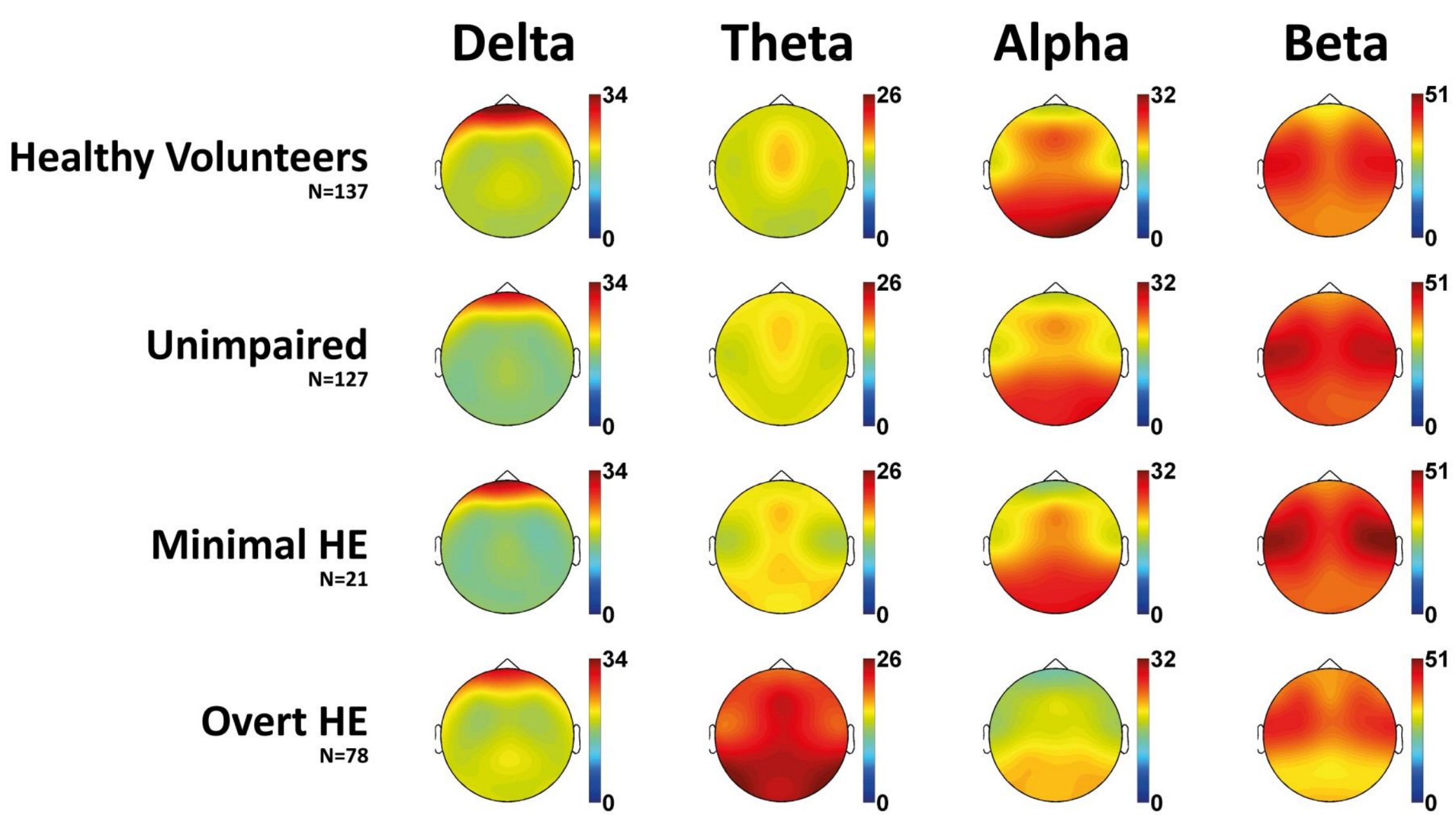

